\title{
Strates
}

STRATES Matériaux pour la recherche en sciences sociales

$8 \mid 1995$

La question de l'environnement: recherches parallèles en Espagne et en France

\section{Gestion du territoire et de l'environnement dans un parc métropolitain. Le parc régional du haut bassin du Manzanares}

Josefina Gómez Mendoza, Carlos M. Manuel Valdés et Ester Sáez Pombo

\section{CpenEdition}

Journals

Édition électronique

URL : http://journals.openedition.org/strates/1017

DOI : $10.4000 /$ strates. 1017

ISSN : $1777-5442$

Éditeur

Laboratoire Ladyss

Édition imprimée

Date de publication : 30 juin 1995

ISSN : 0768-8067

\section{Référence électronique}

Josefina Gómez Mendoza, Carlos M. Manuel Valdés et Ester Sáez Pombo, « Gestion du territoire et de l'environnement dans un parc métropolitain. Le parc régional du haut bassin du Manzanares », Strates [En ligne], 8 | 1995, mis en ligne le 20 décembre 2005, consulté le 08 septembre 2020. URL : http:// journals.openedition.org/strates/1017 ; DOI : https://doi.org/10.4000/strates.1017

Ce document a été généré automatiquement le 8 septembre 2020

Tous droits réservés 


\section{Gestion du territoire et de l'environnement dans un parc métropolitain. Le parc régional du haut bassin du Manzanares}

Josefina Gómez Mendoza, Carlos M. Manuel Valdés et Ester Sáez Pombo

\section{NOTE DE L'ÉDITEUR}

Traduit de l'espagnol par Yves Luginbühl, Aline Brochot et Michèle Petit. Relu par Josefina Gómez Mendoza et Ester Sáez Pombo. (Ce texte a été proposé au VII ${ }^{\text {ème }}$ Colloque de Géographie rurale qui s'est tenu à Cordoue en mars 1994.)

1 Il existe encore peu de travaux sur les résultats de la gestion des espaces naturels protégés. Peut-être parce que l'on ne dispose pas encore d'un temps d'observation suffisant ou d'un dispositif d'analyse approprié, on préfère approfondir l'étude des décisions de protection et de planification et des réseaux régionaux qui en résultent. Cependant, ce sont les actions qui sont réalisées à l'intérieur d'un espace naturel protégé, théoriquement dans une optique de gestion écologique ${ }^{1}$ et en application de son Plan Directeur d'Usage et de Gestion'2, qui ont une traduction dans le paysage et le territoire, et qui en définitive justifient ou non les limites posées à l'activité humaine que la création d'un parc entraîne.

2 Avec environ neuf années d'existence, le Parc régional du haut bassin du Manzanares a été l'objet d'un volume d'actions et de financements suffisamment important pour qu'il soit légitime que nous tentions une première évaluation de la gestion qui a été effectuée - d'autant plus que l'on va procéder à la révision de son Plan Directeur d'Usage et de Gestion ${ }^{3}$. Les actes des réunions de son conseil d'administration et de sa commission permanente, les rapports sur toutes les actions qui ont été réalisées depuis 1985 dans le Parc, les notes et opinions émises par les gestionnaires et les représentants 
des différents secteurs qui composent le conseil d'administration, la documentation cadastrale et les registres de la propriété agraire, en particulier celle des forêts, constituent les bases de l'information qui a été utilisée pour réaliser cette analyse, encore inachevée, et sur laquelle cette communication s'appuie ${ }^{4}$. Car il importe d'apprécier l'évolution du Parc en relation avec les caractéristiques et objectifs qui ont présidé à sa création, en tenant compte de sa situation tout à fait particulière liée à la proximité d'une agglomération de la taille de Madrid. Il convient d'évaluer les processus d'action engagés dans un programme qui vise à concilier la conservation de la nature et le développement des activités et usages traditionnels pour atteindre une complémentarité et une compatibilité satisfaisantes. Il est également fondamental de connaître la politique patrimoniale mise en place et la justification des acquisitions réalisées. Enfin, la gestion du Parc, comme celle de tout espace naturel protégé, pose la question de l'articulation de la planification territoriale et de la planification de l'environnement, d'une part, et de la conservation de la nature et des politiques de développement, d'autre part. Ce sont là les problèmes de toute gestion territoriale et écologique.

Un espace métropolitain convoité, aux valeurs naturelles inégales

3 Les premières tentatives de protection de la Sierra de Guadarrama ont eu lieu dans les années vingt. Après quelques années de débat sur l'opportunité de classer en parc naturel toute la Sierra de Guadarrama, idée défendue depuis Madrid, ou bien de circonscrire la protection aux secteurs de plus grande valeur naturelle, c'est cette seconde option, dont les défenseurs étaient de grands naturalistes participant à

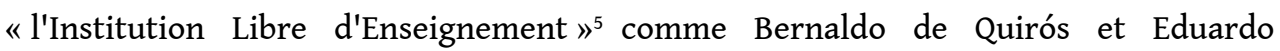
Hernández-Pacheco, qui a prévalu. Ainsi furent classés en 1930 en Sites naturels d'intérêt national une série de secteurs de la Sierra dont faisait partie la Pedriza del Manzanares (Registre officiel du 30 septembre 1930) ${ }^{6}$.

Plus récemment le Décret royal du 4 décembre 1978, également circonscrit au secteur de la Pedriza, procéda à la création du Parc naturel du haut bassin du Manzanares. Les actions ayant pour objectif la protection de cette zone connaissent un tournant décisif avec la promulgation de la loi régionale portant création du Parc régional (Loi 1/1985 du 23 janvier 1985), loi de portée plus large, tant dans ses objectifs que dans son emprise territoriale, que les dispositions citées précédemment. Elle concerne un espace très étendu et très hétérogène. Limités au nord par les sommets de la Cuerda Larga, et au sud par le talus formé par la forêt du Pardo, ces 37364 hectares initiaux incluent une grande variété de paysages : zones urbaines denses, espaces naturels peu dégradés par l'activité humaine et grandes étendues où domine l'activité sylvo-pastorale. ${ }^{7}$. Mais en plus de sa très forte valeur écologique, le caractère métropolitain de cet espace a pesé dans la décision de protection, du fait des risques qu'il impliquait pour sa future conservation. A cet égard, il convient de faire remarquer que l'objectif principal de la loi est « de rechercher un équilibre entre le maintien des systèmes physico-biologiques actuels et le développement des activités économiques et sociales du milieu rural ». Un objectif, en définitive, de gestion intégrée pour lequel la Communauté de Madrid créa ainsi, avec un caractère pionnier, un dispositif de protection, le Parc régional, qui n'existait pas encore dans la législation du moment. Ce fut le principal motif pour lequel le Parti Populaire - qui représente la droite- présenta un recours d'inconstitutionnalité, repoussé par le Conseil constitutionnel le 19 octobre 1989. 
5 Il est plus intéressant de remarquer que cette initiative de création du Parc régional du haut bassin du Manzanares provient du ministère régional de l'Aménagement du territoire (Consejería de Política Territorial), pour lequel il représente un moyen pour gérer les terrains non constructibles soumis à une protection spéciale grâce à un instrument qui vient en complément de la loi foncière. Ainsi, les propositions de la loi précitée comme celles de son Plan directeur dépassent largement ce qui relève strictement de l'environnement, incorporant des compétences plus spécifiques à l'administration sectorielle (agriculture et élevage, sylviculture) et surtout, relevant de l'aménagement du territoire ${ }^{8}$. Ceci explique l'inclusion de certains espaces annexes au bassin du Manzanares (Monte de Viñuelas et, plus tard, Polígono ${ }^{9}$ Valverde) et, également, certains des problèmes qui se sont posés dans la gestion.

Le zonage du Parc : conservation du milieu naturel et développement des activités et usages traditionnels

6 Le zonage établi par la loi fondatrice du Parc est important parce qu'il traduit en grande partie les critères du Plan Directeur d'Usage et de Gestion, et parce qu'il a également des répercussions dans l'administration et la gestion du Parc. Étant donné l'hétérogénéité des paysages, des usages et des états de conservation, et aux fins de "protéger les ressources naturelles de la dégradation ", mais aussi de développer des activités compatibles avec les caractéristiques du milieu naturel (Plan Directeur, pp. 59-60), des zones de niveaux de protection différents ont été délimitées à l'intérieur du Parc. Comme on l'a dit, un des mérites du Plan Directeur est de reconnaitre que ce territoire comprend des espaces très distincts; aussi, pour garantir la viabilité et le succès de la gestion, il convient de tenir compte de la réalité territoriale et d'élaborer " un traitement adapté à chaque type de zone, en évitant autant les excès et contraintes des politiques de conservation classiques dans des zones où leur application serait impossible, qu'un laxisme excessif dans les zones naturelles fragiles et moins menacées par l'homme» (Plan Directeur, p. 61). Le Plan Directeur signale ainsi la possibilité «d'ajuster dans l'espace et dans le temps » les limites établies pour favoriser « une administration adaptée » à tout moment, proposant, en définitive, un aménagement du territoire réaliste et flexible qui garantisse la compatibilité entre conservation et usages traditionnels en tenant compte des pratiques et activités productives existantes dans le Parc. En fonction de ces objectifs le zonage du Parc a été effectué en distinguant les zones suivantes (voir carte) :

7 - Zone de réserve naturelle: Elle comprend des espaces peu modifiés par les activités humaines et du plus grand intérêt "naturel et scientifique». On distingue la Réserve naturelle intégrale (A1) et la Réserve naturelle pédagogique (A2). Les restrictions d'usage sont, comme il est logique, importantes, seules étant permises les activités agricoles, pastorales, forestières et autres activités similaires "répondant à des objectifs de maintien, d'amélioration et de conservation ", ainsi que les activités pédagogiques et de recherche et les activités de loisir comme la randonnée (Plan Directeur, p. 62).

8 - Zones du Parc régional agro-pastoral : Elles sont définies comme celles « qui possèdent une valeur écologique élevée ou moyenne, qui ne comportent pas d'écosystèmes suffisamment préservés, mais qui présentent en revanche des zones de haute ou moyenne valeur productive et comprennent des écosystèmes partiellement modifiés par les usages traditionnels du territoire ». Le Plan Directeur permet et, dans certains cas, favorise, les activités productives à caractère traditionnel, en particulier l'élevage extensif, la pratique de la chasse et de la pêche, et en outre des «usages 
conservatoires " et des "activités éducatives, culturelles et de loisir ", sous réserve qu'elles garantissent la conservation du milieu naturel. A l'intérieur du Parc régional agro-pastoral, la loi fait une distinction entre les terrains à vocation de protection (B1) géosystèmes d'un « haut degré de conservation et de qualité » -, à vocation de production (B2) - zones à grandes capacités de production, mais présentant un degré de conservation moindre - et les zones "à régénérer" (B3) -zones en grande partie dégradées du fait de l'abandon des usages agro-pastoraux traditionnels. Parmi les usages autorisés, citons l'extension de pâturages autochtones dans le premier cas, l'introduction de nouvelles exploitations pastorales et agricoles dans le deuxième, et la reforestation pour la « régénération des écosystèmes » dans le troisième.

- Zones $T$ et $P$ : Enfin, sont définies de façon beaucoup plus restreinte des Zones de transition $(\mathrm{T})$ - bandes situées à l'est et à l'ouest de la lisière de la forêt du Pardo, destinées à la protection de ce domaine et pouvant «recevoir des installations sportives, récréatives et culturelles » sous réserve qu'elles soient compatibles avec sa fonction de protection-, et des Zones à aménager pour la planification urbaine (P), constituées par les noyaux de peuplement et d'urbanisation existant à l'intérieur du Parc. Bien que l'aménagement de ces dernières zones relève des Plans urbains, le Plan Directeur impose certaines restrictions à ces documents, notamment en ce qui concerne la hauteur maximum des nouvelles constructions - «qui ne doivent pas dépasser trois étages, sauf dans la nouvelle ville de Tres Cantos»- et les terrains non constructibles - les zones ainsi qualifiées par la planification urbaine actuelle « devront conserver cette qualification, en raison de leur haute valeur écologique ».

10 Les zones $\mathrm{T}$, et surtout les zones $\mathrm{P}$, constituent une bonne illustration des relations équivoques qu'entretiennent la planification urbaine et la planification de l'environnement à l'intérieur du Parc. Il semble exister une volonté de renforcer les instruments de la loi foncière à travers le Plan Directeur, qui n'a pas empêché les affrontements entre l'administration territoriale, les autorités locales et les gestionnaires du Parc en l'absence d'articulation entre les différents documents qui établissent la destination du sol (normes subsidiaires, PRUG ${ }^{10}$ ). La volonté d'inclure toutes les zones périphériques à l'intérieur du Parc, manifestée dans les extensions successives que nous étudierons dans la partie suivante, constitue une autre forme de recherche de « sécurité » urbanistique par le biais de la protection de l'environnement. La rapide modification des limites d'origine du Parc

11 Bien que l'exposé des motifs de la loi précise que le périmètre du Parc délimite une unité homogène du point de vue de la gestion, et signale en outre que son extension comporterait le risque de «disperser les ressources et d'engendrer des conflits marginaux qui pourraient enlever son efficacité à une gestion de protection et de réhabilitation comme celle qui prétend être entreprise à partir de cette loi $»^{11}$, trois extensions très significatives ont eu lieu au cours des premières années de son application. Ainsi, la Loi du 23 avril 1987 incorpore trois exploitations (Valdelamasa, Las Jarillas et la Dehesa Boyal de San Sebastián de los Reyes, totalisant 1313 hectares) situées au sud de la forêt de Viñuelas, avec un paysage qui comporte des vestiges assez bien conservés de la forêt méditerranéenne; sa proximité de Madrid et la présence d'enclaves en voie de dégradation justifient son inclusion dans le Parc régional de Manzanares.

12 La deuxième extension (Loi du 7 février 1991) affecte le Polígono Valverde (1 459 hectares), territoire localisé entre la forêt du Pardo, la Route nationale 607 et la zone 
urbaine de Madrid. Dans ce cas, l'inclusion dans le Parc de Manzanares est justifiée par la crainte de "pressions spéculatives dans des zones limitrophes à la forêt du Pardo, même si elles sont classées zones non constructibles, qui pourraient mettre en danger leur fonction de zone de transition avec l'espace déjà protégé ». Cette disposition met en évidence le fait que l'on a eu recours à une réglementation de protection de l'environnement, alors que la décision appropriée aurait consisté à garantir le maintien du caractère non constructible que la réglementation urbaine avait attribué à cet espace. La majeure partie de la zone qui est soumise à cette réglementation est qualifiée de zone de transition $(\mathrm{T})$, ce qui la protège de l'urbanisation mais permet, comme nous l'avons dit, l'implantation "d'installations sportives, culturelles et de loisir, et d'infrastructures d'intérêt public et général ».

13 Enfin, la Loi du 4 avril 1991 permet l'incorporation d'une frange localisée à la limite occidentale du Parc, à savoir les exploitations du coto de Pesadilla et la Dehesa de las Pueblas proches de la forêt de Viñuelas. Il s'agit d'une extension importante (près de 7400 hectares), justifiée (sauf dans le cas des deux dernières exploitations mentionnées) par le fait que ces terrains appartiennent au bassin du Manzanares et englobent des espaces de haute valeur écologique, parmi lesquels il faut signaler le Pinar de la Barranca à Navacerrada. Aussi la majeure partie de cette zone a-t-elle été classée zone de réserve naturelle (A1). Mentionnons, au sujet de cette extension, l'opposition initiale de la municipalité de Navacerrada à l'inclusion de son territoire dans le périmètre du Parc, et par ailleurs les suppressions que l'organisme gestionnaire (le conseil d'administration) a opéré dans les propositions initiales présentées par les associations écologistes.

14 Comme conséquence de ces trois extensions, le Parc a vu sa superficie augmenter de 10136 hectares, et atteint aujourd'hui 47500 hectares. Il est intéressant de signaler que les propositions d'extension citées émanent de groupes écologistes de la région représentée dans le conseil d'administration. Elles ont parfois été appuyées par le parti politique Izquierda Unida et par le ministère régional de l'Aménagement du territoire (c'est le cas en particulier du Polígono Valverde). Il reste qu'il existe aujourd'hui une volonté d'en finir avec le "saupoudrage" de ce type d'actions, même s'il faudra certainement inclure dans le Parc la forêt du Pardo.

Extension du patrimoine foncier public du Parc

15 Une des actions les plus remarquables dans la gestion du Parc est, sans aucun doute, l'acquisition d'exploitations agricoles. En effet le Plan Directeur prévoit à cette fin l'attribution de crédits destinés à faciliter une gestion adaptée des espaces protégés, conforme à l'opinion qui était en vigueur il y a plus d'un siècle chez les naturalistes et les forestiers espagnols qui jugeaient nécessaire de donner aux espaces de grande valeur écologique un statut foncier public comme garantie de leur maintien. Il faut signaler à cet égard qu'en 1985 la superficie publique sous tutelle de l'Agence de l'Environnement dans ce secteur était de 6400 hectares, ce qui correspondait à seulement $17 \%$ de la superficie totale ${ }^{12}$. La plus grande partie de ces biens $(86,2 \%)$ sont d'acquisition récente, et correspondent aux achats de l'État réalisés dans le cadre de la politique hydrologique et forestière durant le premier tiers de ce siècle.

16 Ce fut effectivement l'une des zones sur lesquelles des études furent effectuées et des opérations entreprises à des époques déjà anciennes, en relation avec le caractère torrentiel et irrégulier du rio Manzanares dont le cours supérieur comporte le barrage de Santillana, construit pour l'approvisionnement en eau de Madrid. Les études 
réalisées par la quatrième Division hydrologique et forestière au début du $\mathrm{XX}^{\mathrm{e}}$ siècle mirent en évidence la nécessité de reboiser l'amont du bassin du Manzanares, et plus précisément la Sierra de la Pedriza. En vertu du fait que ce secteur fut privatisé pendant la période de vente des biens communaux (à partir de 1855), on considéra qu'il était indispensable d'acquérir ce territoire pour entreprendre un tel reboisement. C'est dans ce contexte que furent réalisés les premiers achats dans le secteur du Soto del Real (Monte Aguirre) et de Manzanares el Real (Hueco de San Blas), achats qui connaîtront leur plus grande intensité après la Guerre civile, lors de la création du Patrimoine forestier de l'État, jusqu'à atteindre en 1985 les 5514 hectares de propriété publique présents dans le Parc (dans les deux territoires municipaux concernés) ${ }^{13}$.

L'article 3.1 de la Loi du 23 janvier 1985 implique la mise en place d'un régime juridique spécial qui stipule que les terrains inclus dans le Parc sont reconnus d'utilité publique, et peuvent faire l'objet d'une expropriation. «La Communauté de Madrid adoptera les mesures nécessaires et instaurera les mesures appropriées afin que les terrains inclus dans le périmètre délimité par la présente Loi et nécessaires pour atteindre ses objectifs, en accord avec les prévisions du Plan Directeur, deviennent des biens publics ». Dans le deuxième point du même article est concédé à la Communauté de Madrid un droit de préemption sur toutes les transactions de biens et de droits des terrains compris dans le périmètre délimité par la Loi. Le Plan Directeur précise les critères qui devront guider l'évolution des acquisitions publiques de terrains : qu'ils soient de préférence limitrophes à d'autres propriétés de l'État ou de la Communauté ; qu'ils présentent des valeurs naturelles remarquables; qu'ils soient inclus de manière préférentielle dans des zones de réserve naturelle; et qu'ils soient d'une superficie suffisante.

Propriétés acquises par la Communauté de Madrid dans le Parc Régional de Manzanares

\begin{tabular}{|l|l|l|l|l|}
\hline Nom & Commune & Superficie & Année & Pesetas/ha \\
\hline \hline El Jaralón & & (ha) & & \\
\hline \hline La Pedriza I & Manzanares el Real & 160 & $1986-88$ & 95000 \\
\hline \hline La Pedriza III & Manzanares el Real & 284 & $1988-89$ & 110000 \\
\hline \hline Cañacerral & Manzanares el Real & 203 & 1988 & $?$ \\
\hline \hline La Pedriza II & Colmenar Viejo & 416 & 1989 & 179104 \\
\hline \hline Hueco de Valdemartín & Manzanares el Real & 289 & 1990 & 131918 \\
\hline
\end{tabular}

Source : Agence de l'Environnement de la Communauté de Madrid

Comme on peut le voir dans le tableau, les achats effectués par l'Agence de l'Environnement sur le territoire du Parc totalisent 1649 hectares, acquis à un rythme régulier entre 1986 et 1990. A l'exception de la propriété Cañacerral de Colmenar Viejo, les acquisitions sont concentrées, comme cela avait été le cas lors de la constitution du 
Patrimoine forestier de l'État, dans la zone de la Pedriza localisée sur le territoire de la commune de Manzanares el Real, ce qui dénote l'intention des gestionnaires du Parc d'accroître le patrimoine foncier public dans des zones de plus haute valeur écologique, désignées par le Plan Directeur comme A1, A2 et B1 ${ }^{14}$.

En ce qui concerne les modalités d'acquisition, l'examen des dossiers montre que, sauf dans le cas de Cañacerral où fut appliqué le droit de préemption stipulé dans les transactions effectuées entre particuliers, les transactions ont été réalisées grâce à des négociations avec les anciens propriétaires, après que ceux-ci les eurent proposé à la vente à la Communauté de Madrid. L'investissement réalisé n'atteint pas le budget prévu pour ces années-là ; cependant les objectifs furent atteints pour les propriétés dont l'achat était souhaité ${ }^{15}$.

La gestion du Parc : priorité à la mise en valeur et à l'ouverture au public

Il est nécessaire d'évaluer la distribution des investissements réalisés depuis la création du Parc pour comprendre les réactions inégales des divers groupes d'acteurs concernés (éleveurs, agriculteurs, chasseurs, propriétaires, etc.) aux restrictions d'usages prévues dans le Plan Directeur.

21 Le tableau suivant résume la distribution des investissements selon les chapitres établis dans le Plan Directeur. Il faut remarquer l'importance du budget attribué à la «mise en valeur agraire ", l'essentiel des ressources financières étant destiné à des travaux d'amélioration des pratiques d'élevage et des pratiques forestières, ce qui traduit bien la volonté de promouvoir les activités économiques traditionnelles de la zone conformément au projet de conservation "active " initial-, comme pour compenser les restrictions d'usage imposées aux habitants de la région. En ce sens, un des acquis de la gestion a sans aucun doute été que l'intérêt témoigné à la population résidente conduise les éleveurs et les agriculteurs à émettre une appréciation favorable.

D'autre part, les financements destinés à « l'accueil et à la promotion » (construction et aménagement de centres de vulgarisation et d'exposition, aménagement d'espaces verts, etc.) sont également considérables; ils doivent permettre de concrétiser la vocation touristique, éducative, culturelle et scientifique de cet espace, et dans le même temps de maîtriser l'afflux massif de visiteurs dans des zones d'intérêt naturel élevé très fragiles (comme la Pedriza).

Investissements par type d'action

\begin{tabular}{|l|l|l|}
\hline Type d'action & Milliers de pesetas & $\%$ \\
\hline \hline Gestion de l'environnement & 322067,1 & 17,9 \\
\hline \hline Mise en valeur agraire & 796600,9 & 44,1 \\
\hline \hline Études et recherches & 32810,5 & 1,8 \\
\hline \hline Accueil et promotion & 356195,7 & 19,8 \\
\hline \hline Information et vulgarisation & 3593,5 & 0,2 \\
\hline \hline Acquisition de propriétés & 118297,0 & 6,6 \\
\hline
\end{tabular}




\begin{tabular}{|l|l|l|}
\hline Gestion de personnel et fonctionnement & 173204,1 & 9,6 \\
\hline \hline TOTAL & 1802768,8 & 100,0 \\
\hline
\end{tabular}

Source : Bordereaux des investissements du Parc, années 1985-1991 (Agence de l'Environnement de la Communauté de Madrid). Les données pour l'année 1989 et pour une partie de l'année 1991, non disponibles à ce jour, n'ont pas été incluses ici. consolidé une identité et une présence motrices dans son territoire, à la fois par l'ampleur et la qualité de ses actions et par sa capacité à se muer en lieu de rencontre et de dialogue entre des intérêts souvent contradictoires. Bien qu'ayant une activité essentiellement réglementaire, le Parc intervient de plusieurs manières dans l'activité locale : il gère les propriétés agricoles de la Communauté, entretient un patrimoine bâti à des fins de loisirs, éducatives et scientifiques importantes, aide financièrement l'élevage et, dans une moindre mesure l'agriculture, réintroduit des espèces végétales et animales, réhabilite des paysages, etc. Ceci confirme les résultats d'autres études similaires, comme celles menées dans le Parc national des Cévennes ${ }^{16}$. Mais à la différence de ce dernier, ce qui, à ce jour, peut être considéré comme le plus grand succès du Parc régional du haut bassin du Manzanares, est sans doute que son activité réglementaire ne soit pas ressentie comme un obstacle au développement local.

Une deuxième conclusion vient préciser et compléter ce qui précède: les objectifs initiaux de développement local fondés sur l'élevage extensif et sur la dehesa ${ }^{17}$ comme mode d'exploitation dominant, parce qu'ils offraient une meilleure réponse écologique et paysagère, ne peuvent être maintenus à cause de la situation difficile du secteur de l'élevage dont l'activité a diminué de moitié durant cette période, d'une part pour des raisons de politique économique générale, et d'autre part du fait de l'abandon de l'activité en raison de la proximité de l'aire métropolitaine. Le Parc devra donc envisager de nouvelles alternatives pour soutenir des activités qui contribuent au maintien ou à la restauration du milieu naturel, comme la chasse.

Enfin, comme on pouvait l'attendre d'un territoire métropolitain, le partage des compétences entre la gestion de l'environnement et la gestion du territoire est encore moins net que ce que l'on trouve habituellement dans les espaces naturels protégés. Nous avons eu l'occasion d'examiner comment dans la création et la délimitation du Parc, la volonté de planification territoriale s'est superposée à la décision de protection de l'environnement. L'histoire de ces neuf années de gestion met en évidence que l'ambiguïté a été la norme, révèle une planification environnementale qui vient au secours de l'aménagement du territoire, et inversement, jusqu'au point où on peut se demander s'il ne s'agit pas d'une ambiguïté volontaire et consentie, même si cela entraîne des dysfonctionnements. En tout état de cause, ces conclusions ne s'appliquent pas à un territoire plus large que celui du Parc. Il serait nécessaire de 
comparer ce qui s'est passé à l'intérieur et à l'extérieur du Parc pour savoir si la gestion environnementale et écologique aboutit à la création d'espaces de réserve, et quels types de comportements, laxistes ou contraignants, sont induits à l'extérieur.

\section{NOTES}

1. Le Programme Causses-Cévennes du PIREN, engagé en 1981 pour étudier le fonctionnement du Parc national des Cévennes en France, sous la direction de Marcel Jollivet, proposa la définition suivante de la gestion écologique : «Gestion dans laquelle les décisions se prennent en tenant compte de leur incidence sur l'ensemble de l'écosystème ; gestion qui s'appuie sur les caractéristiques de l'ensemble de l'écosystème pour accroître sa productivité ». Dans une gestion écologique, les conséquences écologiques (c'est-à-dire sur l'écosystème) d'une modification ponctuelle du milieu induite par l'action de l'homme et, à l'inverse, le système de contraintes que l'écosystème constitue pour l'action de l'homme, sont les paramètres de la décision. Voir Jollivet M.(1983), « Présentation du Programme Causses-Cévennes du PIREN », Études rurales, 89-90-91, pp. 331-333.

2. Le Plan Directeur d'Usage et de Gestion (Plan Rector de Uso y Gestión) est un document qui décrit les différentes modalités de la réglementation, de l'organisation administrative et de la gestion d'un espace protégé. Il a été institué par la Loi nationale 4/89 relative à la «Conservation des espaces naturels protégés et de la flore et de la faune sauvages ». Le lecteur pourra se reporter, dans ce même numéro, à l'article consacré aux « Politiques de protection des espaces naturels : le cas andalou » qui décrit l'ensemble de cette procédure. Nous utiliserons dans cet article la dénomination de Plan Directeur pour y faire référence (NDLR).

3. Le Plan Directeur d'Usage et de Gestion approuvé par le Conseil du gouvernement de la Communauté autonome de Madrid (Ordonnance du 28 mai 1987), sur proposition du conseil d'administration du Parc, avait en principe, une validité quadriennale (1987-1990). En fait cette validité a été prorogée automatiquement jusqu'à aujourd'hui, en ajustant les investissements annuels à l'augmentation du coût de la vie et aux restrictions budgétaires correspondantes.

4. Nous remercions José Manuel Nicolás Zabala, Directeur Conservateur du Parc jusqu'en 1994, pour les facilités qu'il nous a données à tout moment et, en particulier, l'aide qu'il nous a apportée en mettant à notre disposition le fichier des actions et investissements.

5. La Institución Libre de Enseñanza fut créée par Francisco Giner de los Ríos et d'autres universitaires, en réponse à la suppression de fait de la liberté d'expression des professeurs pendant leurs cours par le ministre de l'Instruction publique Orovio qualifiée de "question universitaire ». Initialement limitée à l'enseignement supérieur, elle s'occupa bientôt de tous les niveaux de l'enseignement et de la diffusion de la connaissance scientifique, en préservant son entière indépendance vis-à-vis des instances officielles. La contribution de l'I.L.E. au renouveau de l'éducation, de la 
science, de la culture et de l'éthique, ainsi qu'à l'ouverture de l'Espagne vers l'Europe, fut considérable jusqu'à la guerre civile de 1936-1939 (NDLR).

6. Mollá Ruiz-Gómez M. (1989), El estudio naturalista de la Sierra de Guadarrama. Ciencia, educación y recreo, Thèse de doctorat (inédite), Université Autonome de Madrid, Département de Géographie. Voir en particulier pp. 300-322 sur l'intérêt de l'Institution Libre d'Enseignement pour la Sierra de Guadarrama. Voir du même auteur « El conocimiento naturalista de la Sierra de Guadarrama. Ciencia, educación y recreo ", in Gómez Mendoza J., Ortega Cantero N. (dir.) (1992), Naturalismo y geografía en España, Madrid, Fundación Banco Exterior, pp. 275-346.

7. La plus grande incohérence, en ce qui concerne les limites établies par la loi du Parc, réside probablement dans la non inclusion de la forêt du Pardo - espace remarquable du point de vue naturel, et pour la protection duquel la création du parc avait été envisagée - du fait de l'opposition marquée par son gestionnaire principal, le Patrimoine national, organisme qui gère le patrimoine de l'État, constitué en partie par l'ancien Patrimoine de la Couronne.

8. Il est très significatif à cet égard d'examiner l'argument utilisé par le Conseil du gouvernement de la Communauté de Madrid face au recours déposé par le Parti populaire dénonçant l'inconstitutionnalité de la loi portant création du Parc : « Il ne s'agit pas d'une loi sur les espaces naturels protégés, ni d'une véritable loi de création d'un tel espace (...), mais d'un instrument concret de portée beaucoup plus large, qui n'a pas pour objectif la simple protection de valeurs écologiques ou paysagères expressément désignées, mais l'aménagement et la réglementation des usages (urbains, agricoles, pastoraux et -également- environnementaux) à l'intérieur d'une zone étendue de la Communauté madrilène ", à quoi s'ajoute ultérieurement que " la protection de l'environnement est l'un des contenus nécessaires de l'aménagement du territoire ». En définitive, on argumentait que la loi contestée relevait de la législation de la Communauté autonome en matière d'aménagement du territoire, et non de la législation nationale de protection de l'environnement.

9. Polígono : vaste opération immobilière sur un périmètre défini, assimilable à une zone d'aménagement concerté. (NDLR)

10. Plan Directeur d'Usage et de Gestion. Voir dans ce numéro l'article de Francisco J. Díaz Ojeda et Juan Francisco Ojeda Rivera Politiques de protection des espaces naturels. Le cas andalou. (NDLR)

11. Si on reconnaissait la nécessité d'un traitement spécial des zones limitrophes, leur réglementation se recommandait « d'autres schémas d'aménagement du territoire » (exposé des motifs de la Loi 1/1985 du 23 janvier 1985).

12. Selon les données de l'Agence de l'Environnement de la Communauté de Madrid.

13. Selon les documents des acquisitions conservés par l'ICONA.

14. A la différence de la philosophie sur laquelle se fondent les acquisitions réalisées (toutes concernent des zones de haute valeur écologique), il convient de commenter la tentative d'achat de la propriété appelée Monte Alegre (commune de Torrelodones), soit 9 hectares ceinturés par l'urbanisation dont la propriété fut transférée sans communication préalable au conseil d'administration. Il fut décidé ensuite d'exercer un droit d'examen et de préemption, sans que l'achat soit réalisé (actes du conseil d'administration du 6 février 1988 et du 13 décembre 1993).

15. Dans le Plan Directeur (p. 148) un tableau présente le budget d'investissement pour ce chapitre (325 millions de pesetas) et les propriétés dont l'achat est considéré comme prioritaire : Hueco de Valdemartín, Las Pedrizas et Cañacerral. 
16. Ces résultats sont exposés, notamment dans le tome 5 des Annales du Parc national des Cévennes (1992), où sont présentées les études réalisées de 1981 à 1986 dans le cadre du programme Causses-Cévennes du CNRS (PIREN).

17. Le terme dehesa fait référence à un domaine foncier clôturé, avec une couverture d'arbres plus ou moins dense, qui donne lieu à différentes formes d'exploitation : exploitation forestière (charbon de bois, bois d'oeuvre, autres produits), élevage (pâturages), agriculture (culture céréalière).

\section{RÉSUMÉS}

L'article porte sur la gestion du Parc régional du Manzanares, espace protégé caractérisé par l'inégale valeur naturelle des différentes zones qui le composent, et une forte pression visant à y développer des usages récréatifs et résidentiels, qui tient à sa proximité de l'aire métropolitaine madrilène. Le Parc a réussi à s'affirmer comme unité territoriale de gestion, au point de soutenir l'administration territoriale par la planification environnementale. Toutefois les objectifs de développement local par le biais de l'élevage extensif traditionnel n'ont pu être atteints, du fait de la crise que connaît ce secteur.

Spatial and environment management in a metropolitan park: the Regional Park of the Manzanares upper bassin (Madrid area).

The authors study the management of the Manzanares Regional Park. The preserved area includes a great variety of natural spaces of unequal value, and, due to the proximity of Madrid., it has had to prevent the threatening development of recreation and residential areas. The Park has succeeded in asserting itself as a territorial management unit, in such a way that it has backed the territorial administration by means of environmental planning measures. However, if traditional extensive cattle breeding in the Park was intented to sustain local development, the agricultural crisis has changed the prospect in the matter.

La gestión territorial y ambiental de un parque metropolitano : el Parque Regional de la Cuenca alta des Manzanares (Madrid).

Se revisa la gestión de un espacio protegido, el Parque Regional de Manzanares, caracterizado por el desigual valor natural de sus distintas zonas y la fuerte presión de usos recreativos y residenciales como consecuencia de su cercanía al área metropolitana de Madrid. Se comprueba que el Parque ha conseguido afirmarse como unidad territorial de gestión, hasta el punto de ayudar a través del planeamiento ambiental a la Administración territorial. Sin embargo los objetivos de desarrollo local mediante el aprovechamiento tradicional de ganadería extensiva no se han logrado por la crisis del sector. 
INDEX

Mots-clés : Environnement, Espagne, Gestion de l'environnement, parcs régionaux, Planification territoriale, Manzanares, Aire métropolitaine madrilène

Palabras claves : España, parques regionales, Manejo del medio ambiente, Planificación territorial, Area metropolitana madrileña

Keywords : Spain, regional parks, Environment management, Territorial planning, Metropolitan Madrid area

\section{AUTEURS}

\section{JOSEFINA GÓMEZ MENDOZA}

Professeur d'analyse géographique régionale à l'Université Autonome de Madrid, elle a centré ses recherches récentes sur l'histoire de la politique forestière. Membre du Conseil consultatif de l'Environnement, elle s'intéresse particulièrement à la dimension territoriale de la protection de l'environnement. Elle préside actuellement l'Association des géographes espagnols.

\section{CARLOS M. MANUEL VALDÉS}

Géographe, chercheur au Centre de recherches forestières de l'Institut national de recherches agraires. Ses travaux portent sur l'histoire de la gestion et de l'utilisation des montes publics.

\section{ESTER SÁEZ POMBO}

Enseigne au département de géographie de l'Université Autonome de Madrid. Elle achève actuellement une thèse sur l'histoire des montes publics de la zone septentrionale de la sierra de Madrid du XVIII ${ }^{\mathrm{e}}$ au XX ${ }^{\mathrm{e}}$ siècle. 